\title{
Joint Structure between the Shaft and Cam Lobes in Assembled Camshaft
}

\author{
Zhifang Zhai ${ }^{\text {a }}$, Lianfa Yang ${ }^{\text {* }}$ \\ School of Mechanical \& Electrical Engineering, Guilin University of Electronic Technology, \\ Guilin, Guangxi, 541004, China \\ aZZhifangZhai@126.com, by-lianfa@163.com
}

Keywords: Assembled camshaft, Joint structure, Shaft, Cam lobe, Process, Strength.

Abstract. Assembled camshaft (AC) technology is a type of neo-manufactured technology of camshaft by which components are machined separately and assembled together. Compared with traditional integral-type camshaft, $\mathrm{AC}$ has advantages of lighter weight, material saving, compact structure and excellent performance, etc. Joint structure between the shaft and cam lobes has significant influence on the process and joint strength in AC. In this paper, three familiar joint structures are introduced, and the influences of some parameters on process and strength are analyzed. Then the non-circular joint structures are proposed as outlook of future development.

\section{Introduction}

As one of the three key pairs in engine, a camshaft mainly consists of a shaft, several cam lobes and journal bearings [1]. In practice, every kind of components have various performance requirements. Traditional manufactured technologies of camshaft exist the disadvantages of obvious energy consumption, insufficient material utilization and lower machining efficiency [2]. AC technology makes full use of different materials by performance optimization, so it has advantages of lighter weight, reasonable structure distribution, and lower machining cost, etc $[2,3]$.

The currently joint methods of AC include: interference-fits technologies, expanding technologies and bonding technologies [6]. According to the joint structure used in these joint methods, the structure can be divided into the circular structure, key-groove structures and knurled teeth structures.

\section{Circular Structure}

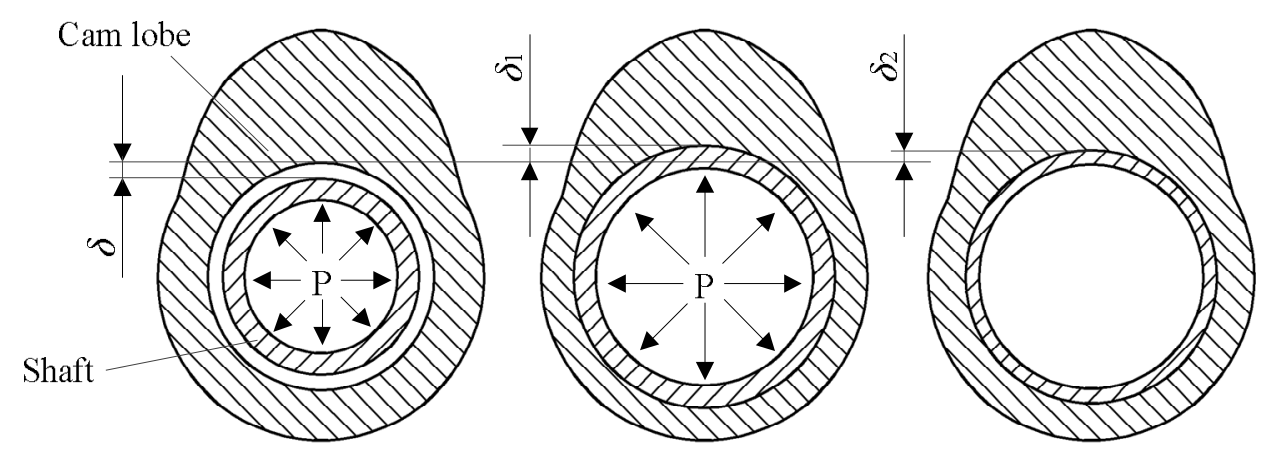

Fig. 1 Schematic drawing of circular structure.

As shown in Fig. 1, there is initial clearance $\delta$ between the shaft and the cam lobe. Shaft and cam lobes are expanded by certain expanding pressure, and the pressure leads to radial displacement on the two components. Joint strength is guaranteed by the joint force between shaft and cam lobes due to the deformation and elastic recovery. The $\delta$ value influences the process and joint strength. Increased $\delta$ value enhances joint strength, but greater $\delta$ value would increase expanding pressure and the difficulty of assembling. However the plastic deformation of cam lobes may occur when it is smaller, debasing the quality of joint structure because of work hardening. In addition, the pronounced stress 
concentration enhances the risk of cracking in $\mathrm{AC}$ [2].

Cam lobes are machined easily by this structure. It has an advantage of saving machining cost, but the lower joint force of this structure is not neglected.

\section{Key-groove Structures}

Key-groove structures can be divided into the spline-groove structures and trapezoidal groove structure.

Spline-groove Structures. The spline-groove structures include structure of involute-spline groove and rectangular spline groove, as shown in Fig. 2. For the former structure, the module of involute is relatively small result from the limited bulging height. The strength of shaft is proportional to the pressure angle $\theta$. Regarding greater pressure angle $\theta$, the positive pressure is improved under the same circumferential load. So it enhances torsion strength, the $\theta$ value is chosen in a high level on the basis of considering performance characteristics of AC. In rectangular spline groove, the groove height $h$ is small due to bulging limitation. The number of teeth correspondingly decreases when the groove width $b$ is large, meanwhile the contour inside cam lobes is similar to round contour and smaller torque resistance occurs. The smaller $b$ value is selected to ensure the number of teeth.

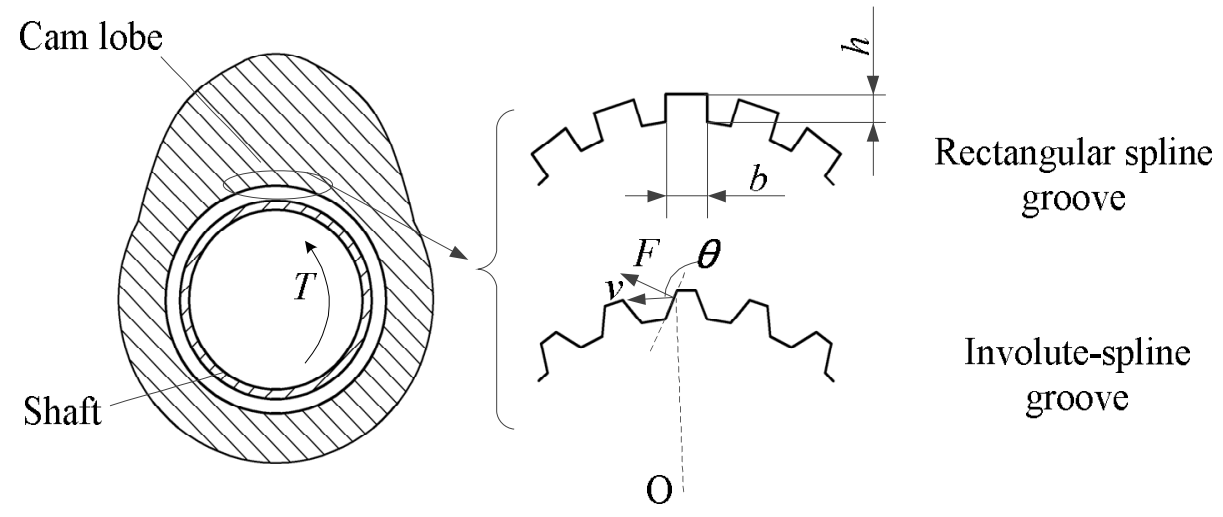

Fig. 2 Schematic drawing of spline-groove structures.

Rectangular spline groove has advantages of higher centring accuracy and reliable stability, but the load resistance is limited for the obvious phenomenon of stress concentration. Although there exists complicated machining process, the better stress distribution and load transmitting determine more applicable use of involute-spline groove in AC.

Trapezoidal Groove Structure. Trapezoidal groove is pointed to the trapezoidal linkage in AC. The surface of shaft is machined to a certain hole shape. Trapezoidal key is pressed into shaft by interference press fitting, and then cam lobes are assembled with them.
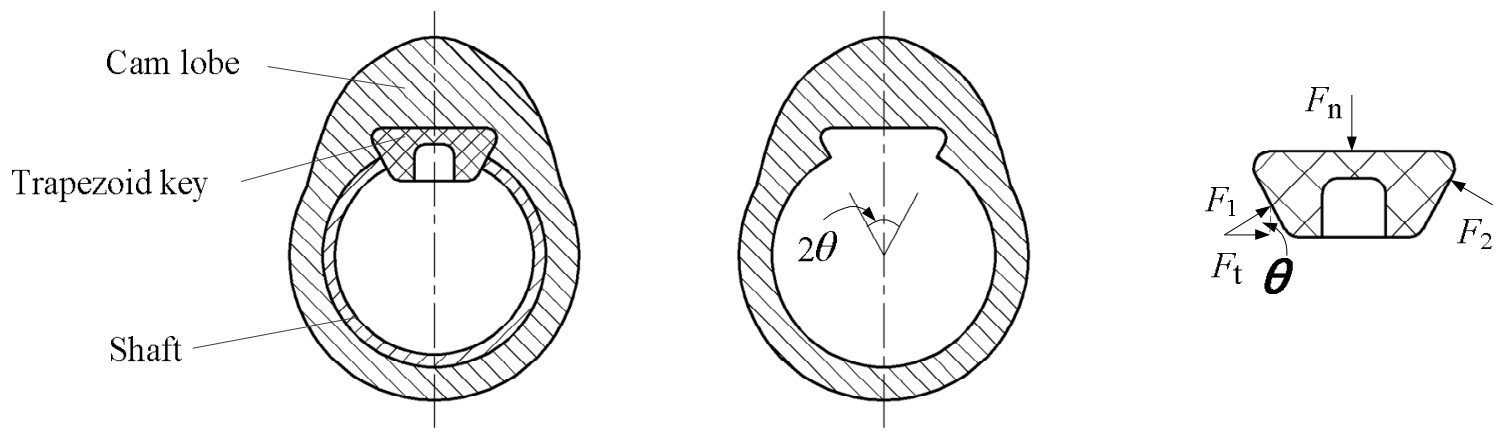

Fig. 3 Schematic drawing of trapezoidal groove structure.

As shown in Fig. 3, trapezoidal key transmits load and torque. While torque is invariant, the shear force $F_{\mathrm{t}}$ in horizontal direction decreases when drafting angle $\theta$ increases. Meanwhile radial extrusion force $F_{\mathrm{n}}$ increases. It has been demonstrated that trapezoidal key is able to transmit larger torque. 
When AC transmits torque exceeding $350 \mathrm{Nm}$, the shear stress is far below the yield strength of material [2]. But at the same time, the cost of fabrication are increased.

\section{Knurled Teeth Structures}

Knurled teeth structures can be divided into the axial and transverse knurled teeth structure.

Axial Knurled Teeth Structure. As shown in Fig. 4, the knurled cutter machines some of teeth along circumferential arrangement. The tooth addendum angle $\theta$ and the tooth height $h$ are determined by cutter. Involute teeth and triangle teeth are mainly geometric shapes of teeth. Qiao et al [4] researched the two shapes, finding that the higher joining strength is obtained by using triangle teeth. The tooth height of cutter determines density of teeth. The greater tooth height could enhance interference, thus increasing torsion strength. But the press-fit load is accordingly larger, and internal residual stress even causes cracking inside cam lobes. Meanwhile it increases assembly load, thereby shortening the life of cutter. So the smaller tooth height is effective to decrease assembly load and prolong cutter life. Increasing addendum angle of cutter enhances assembly inference slightly. The number of cutter teeth greatly affects teeth arrangement, and it should be a multiple of 6 in order to prevent the staggered teeth on shaft in AC.

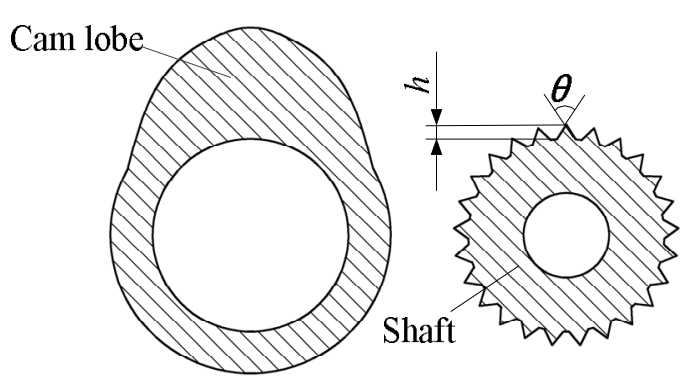

Fig. 4 Schematic drawing of axial knurled teeth structure.

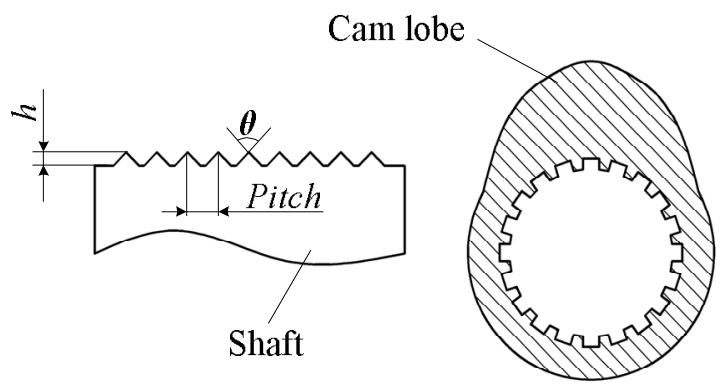

Fig. 5 Schematic drawing of transverse knurled teeth structure.

This structure is suitable for shaft harder than cam lobes. Normally, cam lobes possess wear resistance and pitting corrosional resistance, which limit its application [5].

Transverse Knurled Teeth Structure. As shown in Fig. 5, the transverse knurled teeth processing differs from axial knurled teeth processing. The material on the surface of shaft flows along the axial, and there exists specific shape of shallow grooves inside cam lobes. In the process of joining, the increment of addendum angle of cutter leads to larger tooth width, the same as the press-fit load and torsion strength. The tooth height of cutter should not be too large, otherwise it would weaken torsion strength because of larger tooth width and inadequate material plastic flow. The shallow grooves promote plastic flow of teeth material. However, at present the research about study on shallow grooves is little. It is necessary to carry out relative study on it.

Compared with the axial knurled teeth, transverse knurled teeth is more appropriate because it is suitable for cam lobes harder than shaft. Axial knurled processing is machined conveniently in comparasion with transverse knurled processing.

\section{Conclusions and Outlook}

The model of cam-shaft joint structure is similar to the hub-shaft joint structure except for shafts are thin-walled tubes. Conventional connection is achieved mainly by key and spline. In key connection the keyway branch probably causes stress concentration and reduces fatigue strength. The processing of spline is complex, and it is easily subject to early abrasion and life span reduction if spline is not assembled appropriately [7]. Consequently, some non-circular joint structures are often used to replace the former for uniform stress distribution of the structures. The current contour curves of non-circular structures include: cycloid contour curve, sine contour curve, equidistant polygonal contour curve, 
logarithmic spiral contour curve, etc. Particularly equidistant polygonal contour curve and logarithmic spiral contour curve are most widely used among them. In AC, it is effective to enhance torsion strength by increasing the contact area and the contact friction between the shaft and cam lobes. Press-fit load and torsion strength are main targets evaluating the joint quality of joint structure. They typically have a positive correlation relationship. The joint structure is designed by the two channels: (1) Reducing press-fit load possibly based on torsion strength; (2) Maximizing torsion strength when press-fit load is constant.

\section{Acknowledgements}

The authors gratefully acknowledge the support of the Guangxi Natural Science Foundation (grant number 2013GXNSFAA019305) and Guangxi Key Laboratory of manufacturing System \& Advanced Manufacturing Technology (grant number14-045-15-005Z).

\section{References}

[1] Y.Z. Yang. The research on technology of mechanical expand connection. Changchun: Jilin University, 2010. (In Chinese)

[2] G.M. Han. The assembled camshaft connecting research of tube expanding technology. Changchun: Jilin University, 2012. (In Chinese)

[3] C. Li. Numerical simulation research for knurling process of the assembled camshaft. Changchun: Jilin University, 2013. (In Chinese)

[4] J. Qiao, S.Q. Kou, D.Y. He, S.H. Yang: Materials Science Forum. 575-578 (2008) 216-221.

[5] Z. Peng, S.Q. Kou, H. Zhu, W.C. Liang: Applied Mechanics and Materials. 670-671 (2014) 485-489.

[6] J.T. Chen, L.F. Yang: Advanced Materials Research. 602-604 (2013) 2118-2122.

[7] Y.Y Zheng, J.L. Rao, L. Wu: Applied Mechanics and Materials. 34-35(2010) 1730-1734.

[8] Z.L. Kahn-Jetter, E. Hundertmark, S. Wright: Journal of Mechanical Design. 122(2000) 130-135.

[9] B.Z. Lü, K. Liu: Key Engineering Materials. 460-461(2011) 369-373. 